\title{
Grain Size Determination and Grain Boundary Characterization of Nanocrystalline Thin Films From Conical Dark Field Imaging
}

\author{
A. Darbal*, K. Barmak ${ }^{*}$, T. Nuhfer ${ }^{*}$, T. Sun ${ }^{* *}$, K. R. Coffey ${ }^{* *}$ \\ *Materials Research Science and Engineering Center and the Department of Materials Science and \\ Engineering, Carnegie Mellon University, 5000 Forbes Avenue, Pittsburgh, PA 15213 \\ **Advanced Materials Processing and Analysis Center, University of Central Florida, 4000 Central \\ Florida Boulevard, Orlando, FL 32816
}

Recent studies [1, 2] have identified grain boundaries as the dominant electron scattering sites in the resistivity size effect for $\mathrm{Cu}$. Studies of this kind highlight the growing need for rapid, quantitative methodologies for grain size measurements in nanoscale materials. In this work, we report the use of conical dark field (CDF) imaging obtained by the Automated Crystallography for the Transmission Electron Microscope (ACT) system as an improved method for grain size measurement.[3] Additionally, the ACT technique maps grain orientations, via the indexing of reconstructed diffraction patterns (RDPs), which can then be used to determine grain boundary character (i.e., the five parameters of misorientation axis, angle and grain boundary normal). Thus, ACT not only provides grain size, but also has the potential to examine the impact of different boundary types on the resistivity increase.

The ACT system was used to collect a series of dark field images under different diffraction conditions by the automated tilt and rotation of the beam around the optic axis. The individual, recorded dark field images (e.g., Fig. 1 (a)) were then used to trace the grain boundaries and calculate the grain size for a population of approximately 1000 grains per sample. The samples were four films of $\mathrm{Cu}$ with nominal thicknesses of 30, 35, 40 and $50 \mathrm{~nm}$, deposited onto oxidized $\mathrm{Si}(100)$ wafers with a $150 \mathrm{~nm}$ of thermally grown oxide. The $\mathrm{Cu}$ layers were encapsulated with $20 \mathrm{~nm} \mathrm{SiO}_{2}$ and $2 \mathrm{~nm}$ TaSiN films, forming a $\mathrm{SiO}_{2} / \mathrm{TaSiN} / \mathrm{Cu} / \mathrm{TaSiN} / \mathrm{SiO}_{2}$ structure. The films were annealed at $600{ }^{\circ} \mathrm{C}$ for $30 \mathrm{~min}$ in $\mathrm{Ar}-4 \% \mathrm{H}_{2}$. Average grain sizes were respectively 52.6, 75.1, 92.0, and 114.0 $\mathrm{nm}$.

One advantage of the ACT-CDF method is that individual grains can be very clearly and easily identified, even in the case of our $\mathrm{Cu}$ films where multiple twinning within the grains and lack of adequate grain-to-grain contrast makes grain identification highly problematic in bright-field images (Fig. 1 (b)). Another advantage of the ACT-CDF is that it allows the extent of through-thickness grain overlaps to be quantified without the need for preparation of cross-sectional samples. Knowledge of grain overlaps is important in readily determining the average distance between grain boundaries in the plane of the sample, as is needed for modeling the grain boundary scattering. These two advantages of the ACT-CDF method make it preferable to the hollow cone dark field (HCDF) imaging method employed in [1] and [2] and detailed in [4].

For the development of the methodology for the determination of the boundary type, we have used a $50 \mathrm{~nm}$ thick Pt thin film annealed at $800^{\circ} \mathrm{C}$ for $1 \mathrm{hr}$ (Fig. 1c). We had previously reported the use of the ACT for mapping the grain orientations in this film via indexing of the RDPs.[5] Since this previous report, we have further optimized the data acquisition and indexing procedures and show an example of an improved map in Fig. 1(d). However, specimen drift over the scan time still poses a challenge in that the RDP for a given point is not always from the same grain. We observed that the RDP for a region in the center of the grain is unaffected by specimen drift. Therefore, this 
pattern is used to determine the orientation of the grain and to calculate the distribution of the grain boundary plane normals.
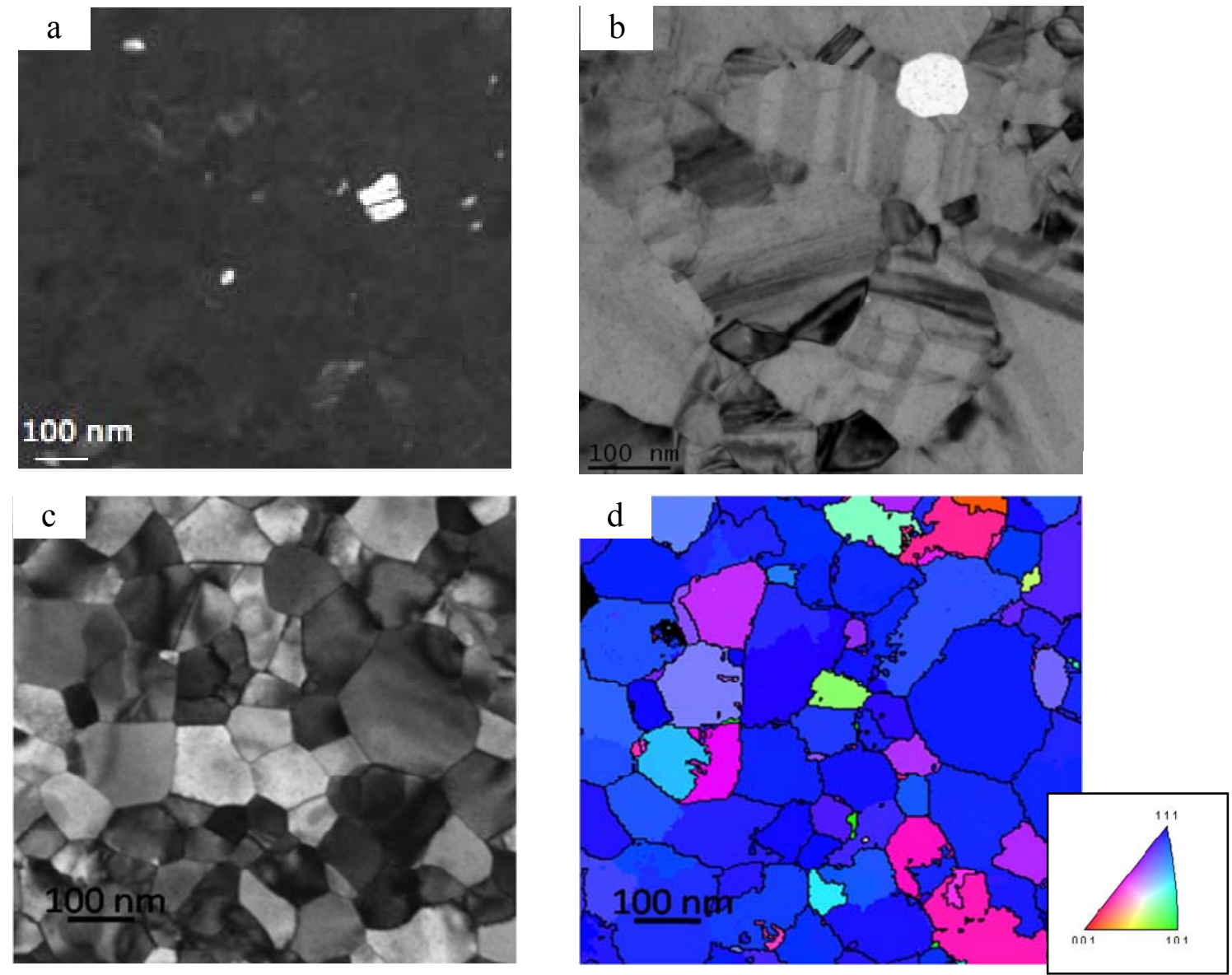

Fig 1: (a) Dark field image of the $40 \mathrm{~nm}$-thick $\mathrm{Cu}$ thin film. White level of this image has been reduced to make grain identification easier, (b) Bright field image for the film in (a), (c) Bright field image of the $50 \mathrm{~nm}$-thick Pt film (d) ACT generated orientation map for the film in (c). Inset gives the color key for the map.

\section{References}

[1] T. Sun, B.Yao, A.P. Warren, V.Kumar, S. Roberts, K. Barmak, K.R. Coffey, J. Vac. Sci. Technol. A 26, (2008) 605, and references therein.

[2] T. Sun, B. Yao, A. P. Warren, K. Barmak, M. F. Toney, R. E. Peale, K. R. Coffey, Phys. Rev. B 79 (2009) 041402(R).

[3] D. J. Dingley, Michrochim. Acta 155 (2006) 19.

[4] B. Yao, T. Sun, A. Warren, H. Heinrich, K. Barmak, K. R. Coffey, Micron 41 (2010) 177.

[5] A. Darbal, K. Barmak, N. T. Nuhfer, D. J. Dingley, G. Meaden, J. Michael, T. Sun, K. R. Coffey, Proceedings of Microscopy and Microanalysis 15 (2009) 1232.

[6] We acknowledge partial support from the NSF MRSEC DMR-0520425 and from the Semiconductor Research Corp., Task 1292.008. S. Levine is thanked for her assistance. 